(5) Except for a few ideal cases, dilatometric studies cannot give unequivocal information on the structure obtained. Nevertheless, this technique is extremely sensitive to any variation in the crystallization process.

\section{ACKNOWLEDGMENTS}

This work was partly supported by a grant from the National Science Foundation. We are much indebted to Professor J. D. Ferry for valuable discussions.

\title{
Origins of Characteristic Bands in the Infrared Spectra of Isotactic Polystyrene and Isotactic Poly (Ring-d ${ }_{5}$ Styrene)
}

\author{
T. ONISHI AND S. KRIMM \\ Harrison M. Randall Laboratory of Pliysics, University of Michigan, Ann Arbor, Michigan
}

\begin{abstract}
The origins of characteristic bands in the infrared spectrum of isotactic polystyrene have been studied with the aid of the spectrum of isotactic poly (ring-d; styrene). It is found that these bands can be classified into three types according to their experimental behavior upon crystallization of the specimen. Assignments show that the bands in any one group are associated with a specific kind of normal vibration, either of the chain or of the benzene ring. Analysis of one of these band types indicates that rotational disordering of the benzene rings may occur, and that crystallization of the polymer is probably correlated with the growth of regions which maintain statistical variability at the local chain level.
\end{abstract}

\section{INTRODUCTION}

A NUMBER of studies on the infrared spectrum of isotactic polystyrene, IPS, has been reported. ${ }^{1-5}$ These indicate that certain characteristic band changes are associated with the transition from the atactic to the isotactic polymer, band changes which are presumably correlated with the appearance of a helical chain conformation in the stereoregular polymer. It appears further $r^{3,4}$ that the spectral changes are of a few distinct types, involving the appearance, frequency shift, or splitting of bands. Band assignment studies on polystyrene have been carried out, ${ }^{6,7}$ and have yielded preliminary information on the nature of the normal modes associated with the above bands. In order to use the infrared spectrum to investigate the physical changes which are associated with the introduction of configurational regularity in a polymer chain, it is clearly desirable to have a more detailed understanding of these band changes. To further this aim, we have undertaken the preparation of isotactic poly (ring- $\mathrm{d}_{5}$ styrene), $\mathrm{IPRd}_{5} \mathrm{~S}$, and the analysis of its infrared spectrum. ${ }^{8} \mathrm{We}$ discuss here the information which this study provides on the origins of the characteristic infrared bands of isotactic polystyrene.

${ }^{1}$ H. Tadokoro, N. Nishiyama, S. Nozakura, and S. Murahashi, J. Polymer Sci. 36, 553 (1959); Bull. Chem. Soc. Japan 34, 381 (1961).

${ }^{2}$ H. Tadokoro, S. Nozakura, T. Kitazawa, Y. Yasuhara, and S. Murahashi, Bull. Chem. Soc. Japan 32, 313 (1959).

${ }^{3}$ M. Takeda, K. Iimura, A. Yamada, and Y. Imamura, Bull. Chem. Soc. Japan 32, 1151 (1959).

${ }^{4}$ M. Takeda, K. Iimura, A. Yamada, and Y. Imamura, Bull. Chem. Soc. Japan 33, $1219(1960)$.

${ }^{5}$ D. Morero, E. Mantica, F. Ciampelli, and D. Sianesi, Nuovo cimento Suppl. 15, $122(1960)$.

${ }^{6}$ C. Y. Liang and S. Krimm, J. Polymer Sci. 27, 241 (1958).

${ }^{7} \mathrm{~S}$. Krimm, Fortschr. Hochpolym.-Forsch. 2, 51 (1960).

${ }^{8} \mathrm{~T}$. Onishị and S. Krimm (to be published).

\section{EXPERIMENTAL}

The isotactic polystyrene used in this study was a commercial sample. It was examined in the quenched state (heated to about $240^{\circ} \mathrm{C}$ and quenched in cold water) and in the crystalline state (obtained by heating at $180^{\circ} \mathrm{C}$ for 30 minutes). Oriented specimens were produced by stretching a film in hot water at $95^{\circ} \mathrm{C}$ over a period of several hours.

The isotactic poly (ring- $\mathrm{d}_{5}$ styrene) was synthesized as follows. Acetic acid anhydride was added to a mixture of benzene- $\mathrm{d}_{5}$ (99\% deuterated) and anhydrous $\mathrm{AlCl}_{3}$ in $\mathrm{CS}_{2}$. The reaction mixture was poured into cold aqueous $\mathrm{HCl}$, and acetophenone- $\mathrm{d}_{5}$ was distilled from an ether extract. This was reduced to the methylphenylcarbinol- $\mathrm{d}_{5}$ by reaction with $\mathrm{LiAlH}_{4}$ in ether. The carbinol was dehydrated to styrene- $\mathrm{d}_{5}$ using activated alumina as a catalyst in flowing $\mathrm{N}_{2}$ at about $300^{\circ} \mathrm{C}$. The resulting styrene- $\mathrm{d}_{5}$ was examined in a mass spectrometer and found to consist of at least $95 \%$ of the ring deuterated species. The styrene- $d_{5}$ was polymerized with a Ziegler type catalyst, ${ }^{9}$ and the isotactic polymer obtained after a methyl ethyl ketone and n-heptane extraction. The melting point of the isotactic polymer was $220^{\circ} \mathrm{C}$.

An x-ray diffraction pattern was obtained of an oriented sample of $\operatorname{IPRd}_{5} \mathrm{~S}$. It is shown in Fig. 1, together with the diffraction pattern of oriented IPS. The fiber axis repeat of the deuterated polymer was found to be $6.65 \mathrm{~A}$. Since this is the same as that of the ordinary polymer, and since the intensity distribution in the diffraction pattern is also essentially the same, we can assume that the structure of IPRd ${ }_{5} \mathrm{~S}$ is the same as the threefold helical structure of IPS. ${ }^{10}$

\footnotetext{
${ }^{9}$ C. G. Overberger and H. Mark, J. Polymer Sci. 35, 381 (1959).

${ }^{10} \mathrm{G}$. Natta, P. Corradini, and I. W. Bassi, Nuovo cimento Suppl. 15, 68 (1960).
} 


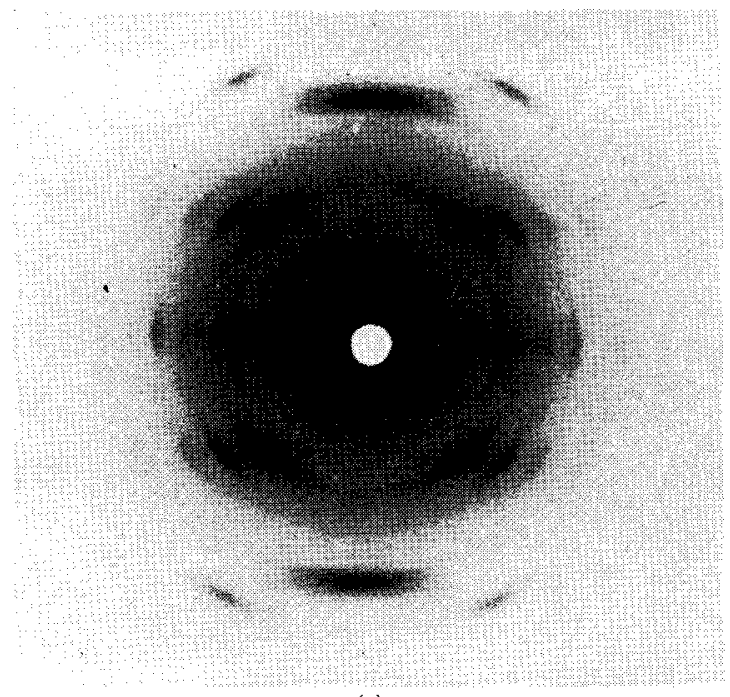

(a)

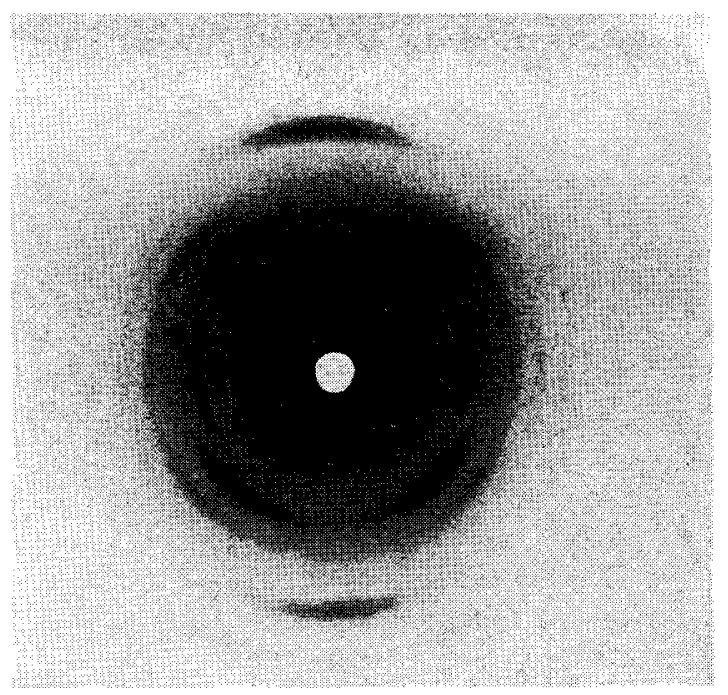

(b)

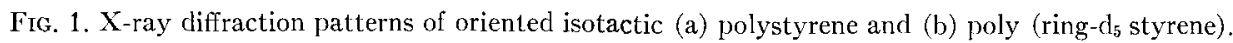

Infrared spectra of quenched, crystallized, and oriented samples of IPS and IPRd ${ }_{5}$ S were obtained with a Perkin-Elmer Model 21 spectrometer with $\mathrm{NaCl}$ and $\mathrm{KBr}$ prisms and a Perkin-Elmer Model 12 spectrometer with $\mathrm{CsBr}$ and $\mathrm{CsI}$ prisms, a $\mathrm{AgCl}$ polarizer being used for the dichroism studies. Spectra of quenched and crystallized polymers are compared in Fig. 2, and polarized spectra are shown in Fig. 3.

\section{RESULTS}

As can be seen from Fig. 2, several characteristic types of band changes occur in the infrared spectra of both
IPS and IPRd $\mathrm{I}_{5} \mathrm{~S}$ when the quenched and crystallized samples are compared. Some of the differences are even more prominent when these spectra are compared with those of the atactic polymers. A classification of the various types of bands which change from the atactic through the crystalline isotactic polymer has been given ${ }^{3,4}$ We have found that this particular classification, while setting out many of the important differences in band behavior, is not entirely satisfactory. Furthermore, we find that a somewhat different basis for classifying these bands is more revealing of their origin. We therefore define the following band classes:
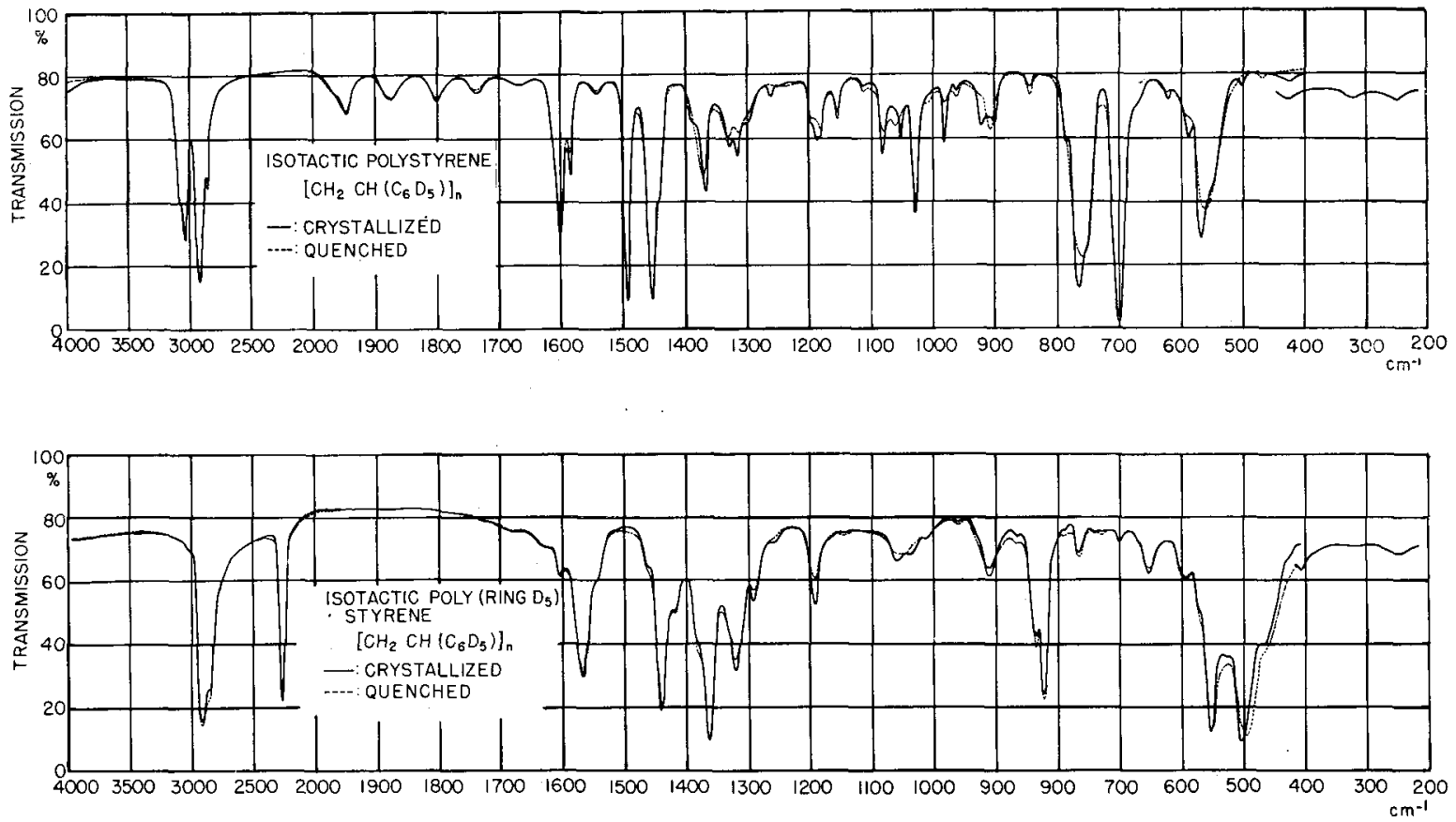

FIG. 2. Infrared spectra of unoriented crystallized and quenched isotactic (a) polystyrene and (b) poly (ring-d $\mathrm{d}_{5}$ styrene). 

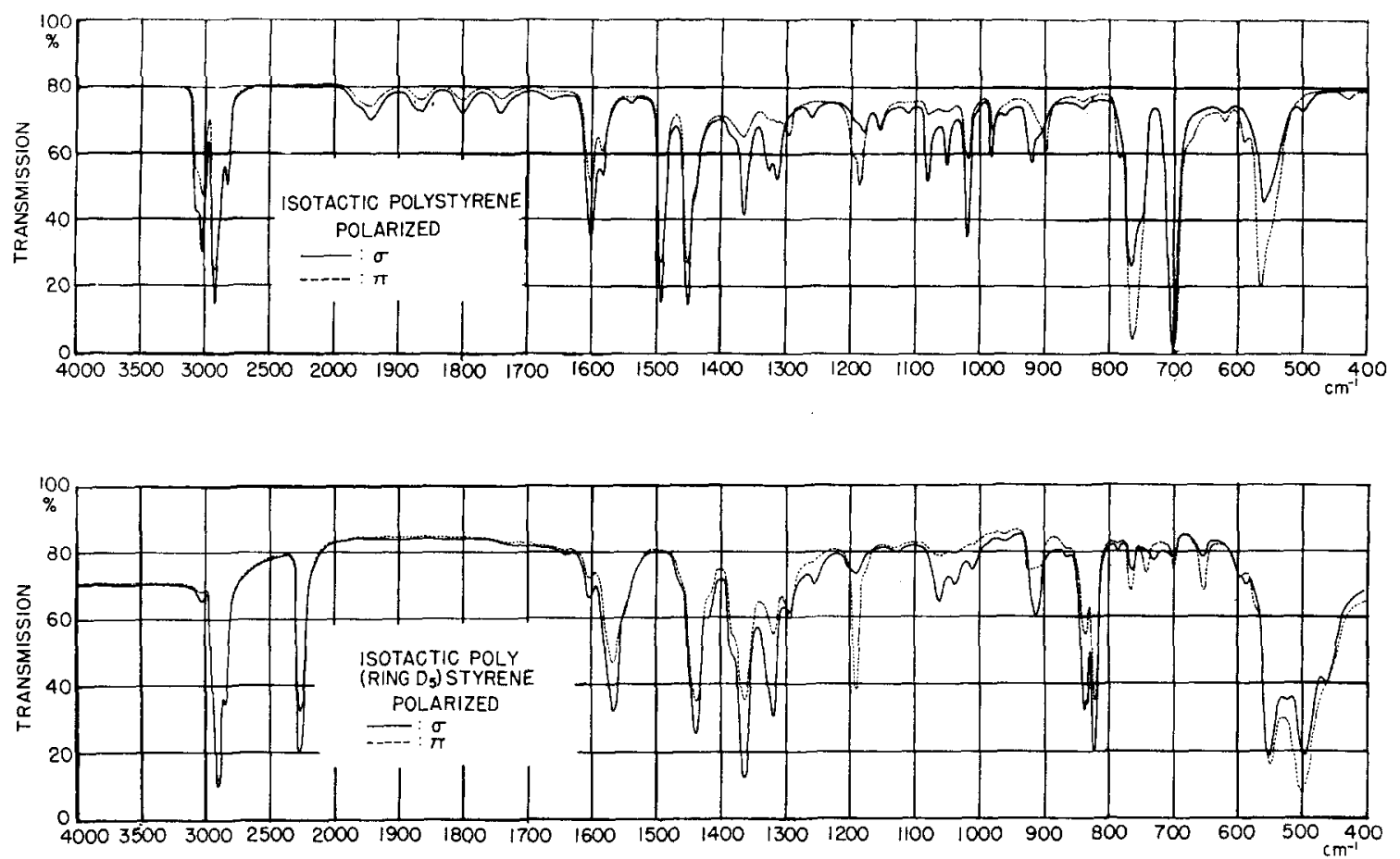

FIG. 3. Polarized infrared spectra of oriented isotactic (a) polystyrene and (b) poly (ring- $\mathrm{d}_{5}$ styrene).

Type I. Bands which are weak or absent in atactic polystyrene, and which increase significantly in intensity in the crystalline stereoregular polymer, in some cases also accompanied by slight frequency shifts. These bands exhibit $\sigma$ or $\pi$ dichroism, with no detectable frequency separation between the $\sigma$ and $\pi$ components of a given band.

TABLE I. Band types and assignments for characteristic bands in isotactic polystyrene and isotactic poly (ring- $\mathrm{d}_{5}$ styrene).

\begin{tabular}{|c|c|c|c|c|c|c|c|}
\hline & \multicolumn{3}{|c|}{ IPS } & \multicolumn{3}{|c|}{ IPRd $\mathrm{d}_{5} \mathrm{~S}$} & Assignment \\
\hline \multirow[b]{2}{*}{ Type I } & $A^{*}$ & $O$ & $C$ & $A$ & $O$ & $C$ & \\
\hline & $\begin{array}{c}1373 \\
\ldots \\
\cdots\end{array}$ & $\begin{array}{c}1370 \\
1298 \\
\cdots \\
\cdots\end{array}$ & $\begin{array}{l}1365 \sigma \\
1297 \pi \\
1260 \sigma \\
1187 \pi\end{array}$ & $\begin{array}{c}1366 \\
1295 \\
\cdots \\
\cdots\end{array}$ & $\begin{array}{l}1365 \\
1291 \\
1253\end{array}$ & $\begin{array}{l}1365 \sigma \\
1293 \pi \\
1265 \sigma \\
1191 \pi\end{array}$ & $\begin{array}{l}\delta(\mathrm{CH}) \\
\gamma\left(\mathrm{CH}_{2}\right) \\
\nu(\mathrm{CC})_{E} ? \\
\nu(\mathrm{CC})_{\mathrm{A}}\end{array}$ \\
\hline \multirow[t]{2}{*}{ Type II } & $1312^{\mathrm{d}}$ & & $1314 \sigma$ & 1050 & & $\begin{array}{l}1061 \sigma \\
1037 \sigma\end{array}$ & $\nu^{\prime}{ }_{3}\left(\mathrm{~B}_{1}\right)_{\mathbf{E}}$ \\
\hline & 1070 & $\begin{array}{l}1078 \\
1060\end{array}$ & $\begin{array}{l}1082 \sigma \\
1051 \sigma\end{array}$ & 837 & & $\begin{array}{l}839 \sigma \\
835 \sigma\end{array}$ & $\nu^{\prime}{ }_{18 \mathrm{~B}}\left(\mathrm{~B}_{1}\right)_{\mathrm{E}}$ \\
\hline \multirow[t]{6}{*}{ Type III } & \multirow{2}{*}{982} & \multirow{2}{*}{982} & $98.3 \sigma$ & \multirow{2}{*}{915} & \multirow{2}{*}{915} & $925 \pi$ & $\nu_{5}\left(B_{2}\right)_{A}$ \\
\hline & & & $\begin{array}{l}982 \pi \\
920 \sigma\end{array}$ & & & $\begin{array}{l}913 \sigma \\
742 \pi\end{array}$ & $\begin{array}{l}\nu_{j}\left(\mathrm{~B}_{2}\right) \mathbf{E} \\
\nu_{17 \mathrm{~B}}\left(\mathrm{~B}_{2}\right)_{\mathbf{A}}\end{array}$ \\
\hline & 906 & 906 & $\begin{array}{l}897 \pi \\
766 \sigma\end{array}$ & 735 & 735 & $\begin{array}{l}730 \sigma \\
655 \pi\end{array}$ & $\begin{array}{l}\nu_{178}\left(B_{2}\right)_{E} \\
{ }_{10 B}\left(B_{2}\right)_{A}\end{array}$ \\
\hline & 757 & 758 & $\begin{array}{l}764 \pi \\
700 \sigma\end{array}$ & 655 & 655 & $\begin{array}{l}654 \sigma \\
553 \sigma\end{array}$ & $\begin{array}{l}\nu_{10 B}\left(B_{2}\right)_{\mathbf{E}} \\
\nu^{\prime}{ }_{11}\left(\mathrm{~B}_{2}\right)_{\mathbf{F}}\end{array}$ \\
\hline & 700 & 700 & $\begin{array}{l}699 \pi \\
566 \pi\end{array}$ & 549 & 553 & $\begin{array}{l}552 \pi \\
501 \pi\end{array}$ & $\begin{array}{l}\nu^{\prime} 11\left(B_{2}\right)_{A} \\
\nu_{4}^{\prime}\left(B_{2}\right)_{A}\end{array}$ \\
\hline & 542 & 558 & $562 \sigma$ & 480 & 496 & $496 \sigma$ & $\nu^{\prime}{ }_{4}\left(\mathrm{~B}_{2}\right) \mathrm{E}$ \\
\hline
\end{tabular}

a $A$ : atactic, $O$ : quenched isotactic, and $C$ : crystalline isotactic a A : atactic, $O:$ quenched isotactic, and $C:$ crystall

c $\nu=$ stretching $\delta=$ bending and $\gamma_{t}=$ twisting.

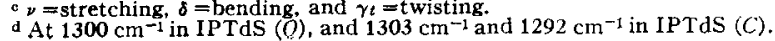

Type II. Bands which do not necessarily increase in intensity, but do split into two $\sigma$ components upon crystallization. Their most important characteristic is that the frequency separation between the components increases with increasing crystallinity of the isotactic polymer.

Type III. Bands which may change slightly in intensity or frequency on crystallization, but whose main characteristic is a splitting into two components, one with $\sigma$ dichroism and the other with $\pi$ dichroism.

The frequency of the bands which fall into these classes are shown in Table I for various states of the two polymers. The assignments indicated in the table are discussed below in terms of approximate normal modes of the polymer chain, those of the benzene ring portion being shown in Fig. 4. The interesting result which emerges from the analysis of the above experimental classifications is that each type of band is associated with a specific group of normal vibrations of the molecule, type I being associated with vibrations of the $\mathrm{CH}$ and $\mathrm{CH}_{2}$ groups and probably also the chain skeleton, type II with the $B_{1}$ species hydrogen ring vibrations, and type III with the out-of-plane modes of the ring.

\section{Type I Bands}

Bands in this class, as we have noted, are characterized by a significant increase in intensity upon crystallization. As will be seen from Table I, they are present in both IPS and IPRd ${ }_{5} S$, thus indicating that they may be correlated with the non-ring portion of the molecule. Assignments for these bands are aided by reference to 
the assignments for polystyrene $e^{7}$ and some of its other deuterated analogues. ${ }^{111}$ Thus, the $1365 \mathrm{~cm}^{-1} \sigma$ band is readily assigned to the $\delta(\mathrm{CH})$ mode, on the basis of its disappearance in isotactic poly- $\alpha \mathrm{d}_{1}$-styrene, ${ }^{11} \mathrm{IP} \alpha \mathrm{d}_{1} \mathrm{~S}$, and its presence in IPRd ${ }_{5} S$. We infer its presence in the latter polymer from the fact that the band at this position, which is expected to arise from the shifted ring vibration at $1452 \mathrm{~cm}^{-1}$ in IPS, is more intense in comparison with the 1440 and $1570 \mathrm{~cm}^{-1}$ bands than is the $1452 \mathrm{~cm}^{-1}$ band in comparison with the corresponding 1493 and $1601 \mathrm{~cm}^{-1}$ bands of IPS. In fact, in IPRd ${ }_{5} S$ there are two components to this band, one at $1365 \mathrm{~cm}^{-1}$ and the other at $1381 \mathrm{~cm}^{-1}$. The $\pi$ band at $1297 \mathrm{~cm}^{-1}$ is most reasonably assigned to $\gamma_{i}\left(\mathrm{CH}_{2}\right)$ on the basis of its position (cf. this mode in polyethylene), ${ }^{7}$ its polarization, and its appearance in the spectra of $1 \operatorname{IPRd}_{5} \mathrm{~S}$ and $\operatorname{IPod}_{1} \mathrm{~S}$, but absence from the spectrum of isotactic poly- $\alpha, \beta, \beta$-tri-deuterostyrene, ${ }^{1}$ IPTdS. It probably shifts to $945 \mathrm{~cm}^{-1}$ in the spectrum of the latter polymer; the shift ratio of 1.37 further supports this assignment, in view of the shift ratios of 1.33, 1.33, and 1.36 for $\nu_{a}\left(\mathrm{CH}_{2}\right), \delta\left(\mathrm{CH}_{2}\right)$, and $\nu_{s}\left(\mathrm{CH}_{2}\right)$, respectively. ${ }^{12}$ The origin of the $\sigma$ band at $1260 \mathrm{~cm}^{-1}$ is not clear. It is present in IPS, IPRd ${ }_{5}$ S (at $1256 \mathrm{~cm}^{-1}$ ), and in IPTdS (at 1250 $\mathrm{cm}^{-1}$ ). It could quite possibly be one of the skeletal modes of the helix. The $\pi$ band at $1187 \mathrm{~cm}^{-1}$ seems quite likely to be assignable to a skeletal vibration on the basis of normal vibration calculations for threefold helical
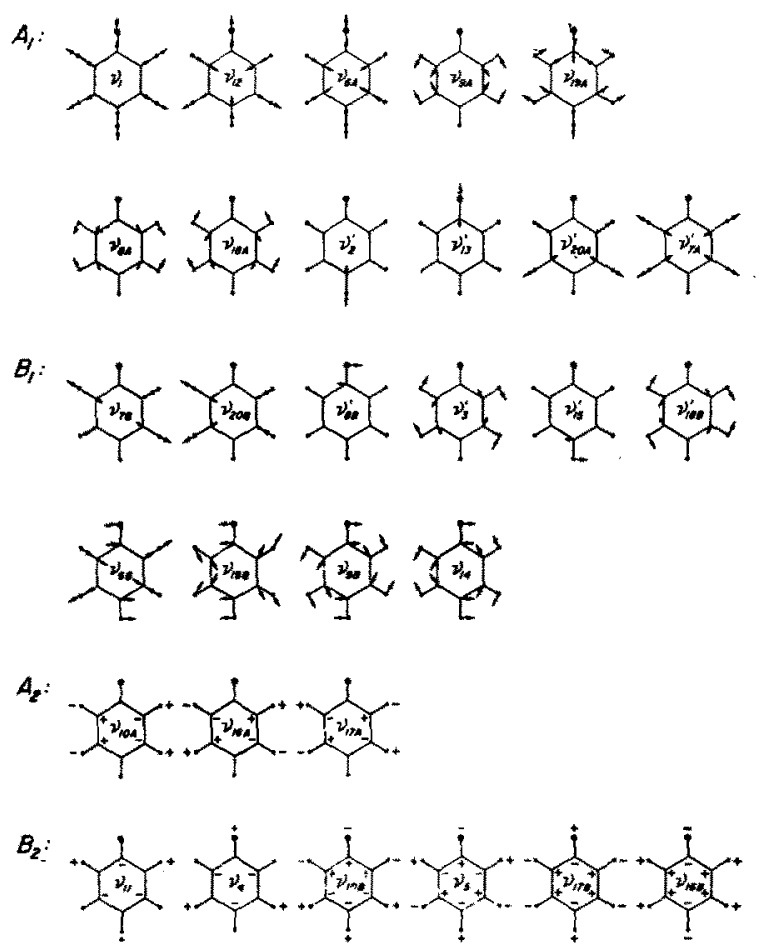

Fro. 4. Approximate normal modes of monosubstituted benzene.

"M. Kobayashi, Bull. Chem. Soc. Japan 33, 1416 (1960).

12 S. Krimm, J. Chem. Phys. 32, 1780 (1960).

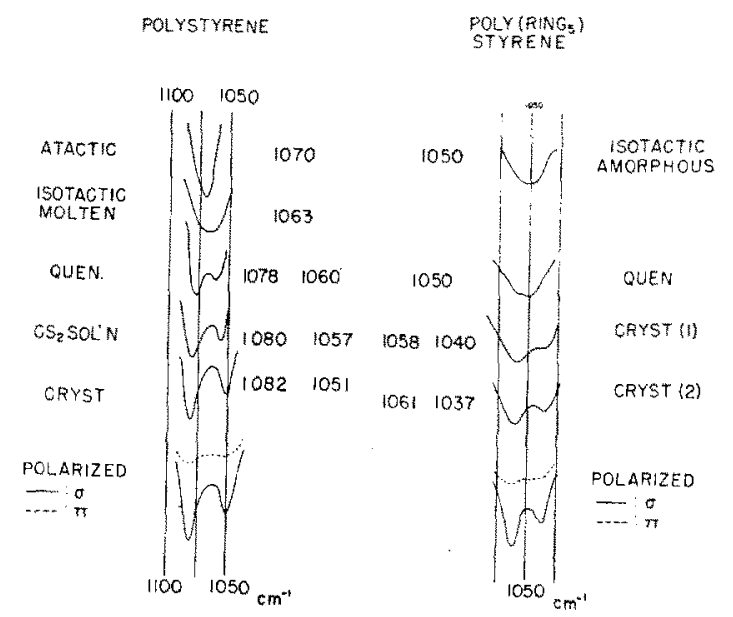

FIG. 5. Splitting in type II bands as a function of crystallinity for isotactic polystyrene and poly (ring- $\mathrm{d}_{6}$ styrene)

vinyl polymers. ${ }^{13}$ Its presence in all of the deuterated species is consistent with this assignment. Its dichroism indicates that it belongs to the A symmetry species of the helical chain. ${ }^{7}$ The type I modes therefore are seen to be correlated with $\mathrm{CH}, \mathrm{CH}_{2}$, and skeletal vibrations.

\section{Type II Bands}

The prime example of a type II band is that at 1070 $\mathrm{cm}^{-1}$ in atactic polystyrene. This band is split in the isotactic polymer, even when in solution. As the extent of crystallization increases, the splitting increases up to a maximum of $31 \mathrm{~cm}^{-1}$. Some of these changes are shown in Fig. 5, where it will be noted that both components show $\sigma$ dichroism. Examination of the spectrum of $\mathrm{IPRd}_{5} \mathrm{~S}$ indicates that these bands have moved to about $837 \mathrm{~cm}^{-1}$; the latter, at 835 and $839 \mathrm{~cm}^{-1}$ in the crystalline polymer, also manifest increased splitting with crystallization. The frequency shift on deuteration is consistent with the assignment ${ }^{7}$ of these bands to the ring mode most closely related to $\nu_{18}\left(\mathbf{B}_{1}\right)$. We have called this $\nu_{18 \mathrm{~B}}^{\prime}\left(B_{1}\right)_{\mathrm{E}}$, where the subscript $\mathrm{E}$ indicates that it belongs to the $\mathrm{E}$ symmetry species of the helical chain. ${ }^{7}$ As will be seen from Fig. 5, the spectrum of $\mathrm{IPRd}_{0} \mathrm{~S}$ shows a group of bands of similar behavior near $1050 \mathrm{~cm}^{-1}$. We believe that this group can be traced to bands near $1300 \mathrm{~cm}^{-1}$ in IPS. In IPTdS there is a band at $1300 \mathrm{~cm}^{-1}$ in the quenched material which begins to split into components at 1303 and $1292 \mathrm{~cm}^{-1}$ upon crystallization. ${ }^{1}$ In IPS these bands are not as clearly defined, but it appears that the band at $1312 \mathrm{~cm}^{-1}$, moving to $1314 \mathrm{~cm}^{-1}$ on crystallization, can be correlated with this group. The situation is complicated somewhat by the presence of the $1328 \mathrm{~cm}^{-1}$ band, which seems to correlate with the $1330 \mathrm{~cm}^{-1}$ band of IPTdS. Our interpretation is that these two bands are associated with the $\nu_{3}$ and $\nu_{14}$ modes, found at 1350 and $1309 \mathrm{~cm}^{-1}$,

1s T. Onishi and S. Krimm, Symposium on Molecular Structure and Spectroscopy, Columbus, Ohio, June, 1961. 
respectively, in benzene, ${ }^{14}$ and that whereas the ring deformation mode $\left(\nu_{14}\right)$ moves from $1330 \mathrm{~cm}^{-1}$ to 1320 $\mathrm{cm}^{-1}$ (in IPRdis), the hydrogen deformation mode $\left(v_{3}\right)$ moves from about $1300 \mathrm{~cm}^{-1}$ to $1050 \mathrm{~cm}^{-1}$ (in $\operatorname{IPRd}_{5} \mathrm{~S}$ ). In IPS there may be some interaction between these various vibrations which results in a slight change in these frequencies, viz., to 1328 and $1312 \mathrm{~cm}^{-1}$. The type II bands therefore arise from the $B_{1}$ species hydrogen ring modes and result in $\mathrm{E}$ species chain modes which split progressively on crystallization.

\section{Type III Bands}

The bands which are listed in Table I under type III all exhibit the characteristic of splitting into $\sigma$ and $\pi$ pairs upon crystallization. They have been assigned ${ }^{7}$ to out-of-plane ring modes, and the frequency shifts observed in going from IPS to IPRd $_{5} \mathrm{~S}$ bear out these assignments. It should be observed that whether the modes indicated by a prime are as shown in Fig. 4 or are closer in form to the (unprimed) modes of benzene cannot be ascertained at present. The modes shown in Fig. 4 represent only a plausible approximation to the possible forms of monosubstituted benzene modes. The splitting of these bands indicates that for the $B_{2}$ ring modes the interaction between units along the helix is sufficiently large to give different frequencies for the A and the $\mathrm{E}$ chain modes. This was not observed to be true of the types I and II bands.

\section{DISCUSSION}

These results raise several questions about the underlying basis of the infrared spectral changes, to which only partially complete answers can be given at present. A detailed understanding will result only from a complete vibrational analysis of the polystyrene spectrum. We will attempt here to consider only the possible plausible bases for the observed effects.

Before doing this, it is necessary to consider the kinds of changes which might be expected in the infrared spectrum as a result of a change from an atactic to an ordered isotactic polymer. These can be of three kinds. First, there may be configurational isomer effects, such as gauche or trans configurations about neighboring bonds, which influence the local normal mode of a portion of the molecule. This could give rise to frequency shifts, intensity changes, or to the appearance of new bands. Second, the isotactic configuration could result in the formation of a regular chain structure, such as the threefold helix in the present case, whose symmetry then affects the spectrum. For example, the interactions between monomer units in one repeat of the helix can give rise to splittings in some of the normal frequencies and to the appearance of additional skeletal vibrations. It should be noted that this corresponds to a kind of one-dimensional crystallization and could quite con-

${ }^{14}$ S. Brodersen and A. Langseth, Mat. Fys. Skr. Dan. Vid. Selsk. 1, 1 (1956). ceivably occur in solution or in a quenched polymer sample over short lengths of the chain. Third, if the increased one-dimensional order permits the growth of three-dimensionally ordered, or crystalline, regions, then interchain interactions may occur with effects somewhat similar to those for the one-dimensionally ordered case. The problem is to distinguish between these effects in the case of polystyrene.

The behavior of the type III bands upon crystallization is most readily understood in terms of the formation of helical chain segments. For such structures we expect ${ }^{7}$ that the normal vibrations will divide up into $\mathrm{A}$ species with transition moment parallel to the helix axis, and $\mathrm{E}$ species with transition moment perpendicular to the helix axis. If the interaction between units along the chain is large enough, the $A$ and $E$ chain modes corresponding to a given normal mode within one unit will occur at slightly different frequencies. This appears to happen for the out-of-plane type ring vibrations in polystyrene, although why it is observed for these modes and not any others is not completely clear. It probably implies that interaction for such essentially out-of-plane modes is greater between units in the chain than for the other types of normal vibrations of the monomer unit.

It is important to note in this connection that not all of the type III bands, although associated with out-ofplane ring modes, must be thought to be pure benzene ring modes. The evidence indicates that many of them are probably mixed in nature. For example, although the 700 and $757 \mathrm{~cm}^{-1}$ bands of IPS are well assigned to $\mathrm{B}_{2}$ species ring modes, they nevertheless show different dichroic ratios. Measurements which we have made on thin oriented films, about 0.0002 in. thick, show that the parallel dichroism of the $757 \mathrm{~cm}^{-1}$ band is greater than that of the $700 \mathrm{~cm}^{-1}$ band by a factor of about 1.85 . Since the orientation distribution cannot produce such an effect in a single sample, it follows (unless the bands are composite, for which there is no evidence) that the transition moments of the two vibrations involved cannot be exactly parallel to each other. The mixed character of some of these out-of-plane vibrations may account for the fact that, although all $\mathrm{B}_{2}$ ring modes should give rise to stronger $\pi$ than $\sigma$ absorption, this is not the case for two of the bands, viz., those at 982 and at $906 \mathrm{~cm}^{-1}$.

In the case of the type I bands, in which significant intensity changes occur on crystallization, the origin of the changes is not in all cases clear. Of course, for the skeletal modes associated with the helical structure the enhanced intensity of such bands in the more highly crystalline material is understandable. The $1187 \mathrm{~cm}^{-1}$ band and possibly that at $1260 \mathrm{~cm}^{-1}$ appear to belong in this category. The origins of the intensity changes in the 1365 and $1297 \mathrm{~cm}^{-1}$ bands are less certain. These effects may arise from the altered form of the normal mode in the helical chain or from a general environmental effect involving coupling with other vibrations. There is no evidence to suggest that these bands are 
associated with local isomeric forms of the groups involved.

The behavior of the type II bands is the most puzzling of all. The symmetry analysis ${ }^{7}$ would allow a progressive splitting of a band with, for instance, increasing helix length, but only into $\mathrm{A}$ and $\mathrm{E}$ modes, i.e., one $\pi$ and one $\sigma$ component, not into two $\sigma$ bands. The latter might occur if another normal mode associated with the helix were to interact with the $B_{1}$ ring modes to which we assign these bands. However, both on the basis of the shift of the $1070 \mathrm{~cm}^{-1}$ doublet to a doublet at $8.37 \mathrm{~cm}^{-1}$ and the existence of two such groups of bands in different frequency regions, we are inclined to discount this explanation. Another possibility is that the splitting arises from interaction between neighboring chains. In the determination of the crystal structure ${ }^{10}$ it has been noted that a statistical variability in chain conformation and direction may occur such that neighboring chains are or are not related to each other by a center of symmetry. This would suggest the possibility of different frequencies resulting from the distinct interactions associated with these two locally different structures, perhaps accounting for the observed splitting. The difficulty with this explanation is that we are hard pressed to account for the large splitting (about $23 \mathrm{~cm}^{-1}$ at $1070 \mathrm{~cm}^{-1}$ ) observed in $\mathrm{CS}_{2}$ solutions of IPS, ${ }^{4}$ where we presume that side-to-side aggregation of helical regions is not extensive.

We wish to suggest what seems to be a plausible explanation of this splitting. The existence of degenerate $E$ species helical chain modes is a consequence of the threefold screw $\left(\mathrm{C}_{3}\right)$ symmetry of the structure. If this symmetry were relaxed or destroyed, then the degeneracy of these modes would be removed. The result could be that a band associated with a given $\mathrm{E}$ species mode of the perfect helix would split into two $\sigma$ components of slightly different frequency. The most likely mechanism for the relaxation of the perfect $\mathrm{C}_{3}$ symmetry is the rotational disordering of the benzene ring about the bond connecting it to the chain. Steric considerations ${ }^{10,15}$ indicate that the favored position is such that the plane of the benzene ring bisects the $\mathrm{CCC}$ angle of

${ }^{15}$ C. W. Bunn and E. R. Howells, J. Polymer Sci. 18, 307 (1955). the chain at the carbon atom where it is attached. It is possible, however, that other orientations of this plane are permissible. In particular, in the statistically variable crystal structure ${ }^{10}$ in which a given chain can be surrounded by either of two types of chains, it is possible that two different orientations of the benzene ring about its local symmetry axis (the bond connecting it to the chain) could occur. This would destroy the $\mathrm{C}_{3}$ symmetry for certain of the ring modes, thus leading to the splitting of the originally degenerate mode. It is interesting to note that the symmetry of the remaining portion of the chain is unaffected by this rotational disorder, so that we do not anticipate splittings for $\mathrm{CH}, \mathrm{CH}_{2}$, and skeletal modes. Furthermore, the $\mathrm{C}_{3}$ symmetry is essentially preserved for the $A_{1}$ species ring modes, whose transition moments are parallel to the symmetry axis of the benzene ring, so that we would not expect splittings to occur for these vibrations. The effect should be maximal for the $B_{1}$ species ring modes, where the transition moment is perpendicular to the symmetry axis of the ring. While this rotational disordering may be a maximum in the statistically variable crystal, it probably would also occur to some extent (perhaps smaller) in the isolated chain. We think that this could account for the observed splittings in the quenched polymer and in solution (cf. Fig. 5), the smaller magnitudes of the splittings in these cases being related to the smaller departure from extended $\mathrm{C}_{3}$ symmetry in the helical portions of the chain. If this mechanism accounts for the progressive splittings in the type II bands, then it suggests that enhanced crystallization of the polymer is not associated with a growth of regions of specific space group symmetry (the $R \overline{3} \mathrm{c}$ and $\mathrm{R} 3 \mathrm{c}$ symmetries allowed by the crystal structure), ${ }^{10}$ but rather the extension of regions which locally maintain the statistically variable chain structure.

\section{ACKNOWLEDGMENTS}

Support is gratefully acknowledged from the U. S. Public Health Service and the National Science Foundation. We are indebted to Dr. W. Burlant of the Ford Motor Company Scientific Laboratory for assistance in carrying out the polymerization of the ring-deuterated polystyrene. 